\title{
An Open-Label, Single Center, Retrospective Study to Evaluate Clinical Outcomes with Surgical Sealant in Bentall Procedures: A Cohort Study
}

\author{
Jiawei Gu, MD, Hao Lai, MD, PhD, Jun Li, MD, Yongxin Sun, MD, Chen Liu, MD, Yulin Wang, MD, \\ Zhiqi Zhang, MD, Le Kang, MD, Ben Huang, MD, Chunsheng Wang, MD
}

Zhongshan hospital affiliated to Fudan University, No. 180, Fenglin Road, Xuhui District, Shanghai City 200032, China

\section{ABSTRACT}

Background: Cardiovascular surgery is associated with substantial risk for postoperative bleeding with increased patient morbidity and mortality. Numerous intraoperative techniques have been utilized to reduce this risk. This study was to assess postoperative bleeding-related parameters following Bentall procedures and to examine the impact of intraoperative surgical sealant application.

Method: The medical/surgical records of 100 consecutive Bentall procedure cases were examined retrospectively for perioperative surgical sealant use and postoperative bleedingrelated outcomes.

Results: Of the 100 patient cases, three died during the postoperative period, and 97 were evaluable. Surgical sealant was utilized in 56 patient cases $(57.8 \%)$. The utilization versus no utilization of surgical sealant was associated with significant reductions in most postoperative bleeding-related parameters, including less drainage $(P=.028)$, resternotomy for hemorrhage $(P=.036)$, transfusion of red blood cells $(P=.022$ at 48 hours; $P=.027$ total in-hospital), transfusion of fresh frozen plasma $(P=.04$ at 48 hours; $P$ $=.004$ total in-hospital), and a higher percentage of cases not needing blood transfusion $(P=.002)$. The surgical sealant group had longer cardiopulmonary bypass circuit $(P=.016)$ and aortic cross-clamp time $(P=.001)$, with no significant between-group differences in intubation time $(P=.636)$ or intensive care unit duration $(P=.294)$. When excluding urgent cases or Stanford type A aortic dissections, intensive care unit duration significantly was shorter in the surgical sealant group $(P=.017)$. Surgical sealant use was not associated with any adverse events.

Conclusion: The application of surgical sealant to the anastomosis suture line in Bentall procedures reduces postoperative drainage, bleeding, and transfusion utilization. Further studies are warranted to investigate these benefits in prospective, randomized clinical trials.

Received February 11, 2019; received in revised form August 28, 2019; accepted September 11, 2019.

Correspondence: Chunsheng Wang, MD, Zhongshan Hospital Affiliated to Fudan University, No. 180, Fenglin Road, Xuhui District, Shanghai City 200032, China; +86-21-64041990-2512 (e-mail: wang.chunsheng@zs-hospital.sh.cn).

\section{INTRODUCTION}

Cardiovascular operations are associated with high rates of perioperative bleeding and often require blood transfusion(s). Data from the Society of Thoracic Surgeons (STS) Adult Cardiac Surgery Database (ACSD) indicates that $50 \%$ patients of cardiac procedures receive blood transfusion, with aortic aneurysm having a rate of $54 \%$ and lower rates generally observed with coronary artery bypass graft (CABG) [Robich 2015]. Among cardiac surgical procedures, that of aortic surgery carries a substantial risk for bleeding [Velasquez 2017].

In the United States, the STS ACSD reported that approximately 290,000 adult cardiac procedures were performed in 2016 , with $5 \%$ of these $(\sim 14,500)$ being aortic aneurysm procedures [D'Agostino 2019]. In China, over 218,000 cardiovascular procedures were performed during 2016, with this number excluding data from Hong Kong and Taiwan. Among the procedures performed in China, the majority $(72 \%)$ required cardiopulmonary bypass $(\mathrm{CPB})$ and $~ 7 \%$ were aortic procedures [Hei 2017].

Perioperative bleeding and the need for transfusion in those undergoing cardiovascular procedures impart substantial morbidity and mortality risks to patients and in the utilization of healthcare resources. Studies have shown that bleeding at vascular anastomoses lengthens operating time and increases blood transfusion requirements as well as increases the risk of re-thoracotomy and lengthens hospital stay [Milne 1996; Geissler 2015]. As a result, techniques and products to reduce both the risk of bleeding as well as transfusion use are the focus of many cardiovascular surgeons. In particular, the use of hemostatic agents and polymeric surgical sealants have been demonstrated in guidelines to adhere to vascular anastomoses and seal potential bleeding sites through cross-linking [Ferraris 2011; Association of Perioperative Registered Nurses 2013].

The surgical sealant, CoSeal (Baxter Healthcare Corp., Deerfield, IL, USA), is composed of two synthetic polyethylene glycols (PEGs), a dilute hydrogen chloride solution and a sodium phosphate/sodium carbonate solution. It is indicated for sealing suture lines along arterial and venous reconstructions to prevent/reduce postoperative bleeding by mechanically sealing. It has been applied to anastomotic suture lines with the intent to reinforce it and to prevent perioperative hemorrhage and the need for blood transfusions. The use of CoSeal has been reported to be effective for managing 
anastomotic bleeding after implantation of Dacron grafts during aortic reconstruction for non-ruptured aneurysms [Hagberg 2004]. Several other studies also have reported that the use of the polymeric surgical sealant for reinforcement along anastomotic suture lines in aortic root reconstruction is effective in bleeding prevention [Dapunt 2008; DeAnda 2009; Glickman 2002].

To our knowledge, there have been few systematic reports on the use and outcomes with polymeric surgical sealants in patients undergoing cardiovascular surgery, specifically Bentall procedures, in China. The objective of this retrospective analysis was to examine postoperative bleeding-related parameters and outcomes in patients who had undergone Bentall procedures in which polymeric surgical sealant was applied to the anastomotic suture line and compare these with cases in which surgical sealant was not used. Also, we describe the technique that we utilize in the application of surgical sealant during these procedures.

\section{MATERIALS AND METHODS}

The surgical and hospitalization records of 100 consecutive patients, who underwent Bentall procedures in the Cardiac Surgery Department in a large hospital in China between January 2013 to December 2013, were reviewed.

Initial data collected from the review included patient demographics, indication for surgical intervention, and perioperative utilization of surgical sealant. Overall and bleedingrelated outcome data collected included operative outcomes (e.g., CPB time, aortic cross-clamp (ACC) time), postoperative outcomes (i.e., requirements for red blood cells and fresh frozen plasma transfusion, postoperative blood loss, rethoracotomy for postoperative hemorrhage, intubation time, and intensive care unit (ICU) duration), and mortality. Postoperative blood loss was defined as drainage volumes within 48 hours following the procedure.

The patient-case demographics and overall- and bleedingrelated outcome data were compared between the patient cases in which surgical sealant was applied to the anastomotic suture lines of the aortic root reconstruction versus those in which surgical sealant or any other additional hemostatic agent was not utilized. The work has been reported in line with the STROCSS criteria [Agha 2017]. Statistical analysis was performed with the IBM® SPSS ${ }^{\circledR}$ Statistics 20.0 statistical software package (IBM corp., Armonk, NY, USA). Continuous variables were expressed as mean \pm standard deviation (SD), and the comparison between two groups variables were analyzed with Student's t-test. Otherwise categorical variables were analyzed with the Mann-Whitney $U$ test or Fisher's exact test, where appropriate. A $P$-value of $<.05$ was considered statistically significant.

\section{RESULTS}

Among the 100 patient cases that were reviewed, three died within 48 hours postoperatively due to multiple organ

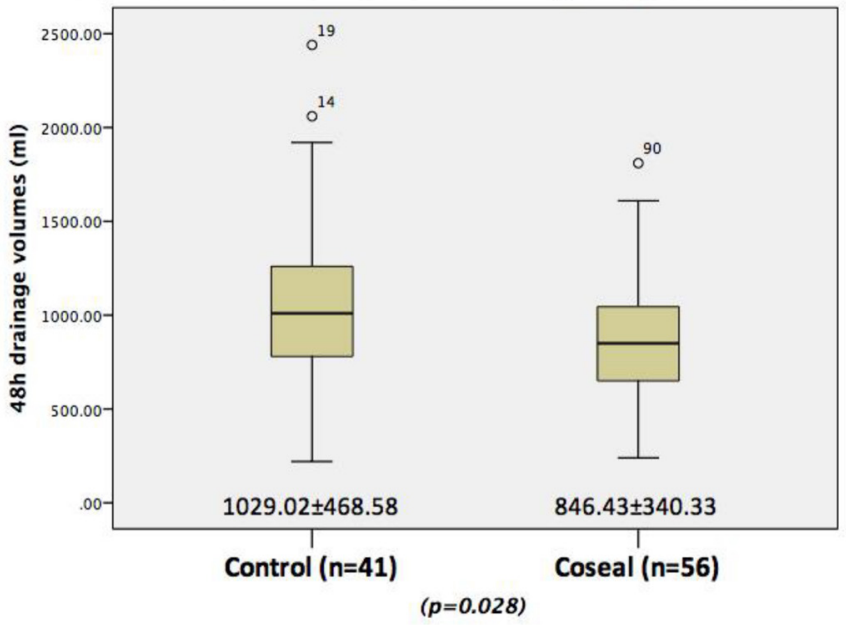

The postoperative blood loss is defined as drainage volumes within 48 hours following the procedure.

dysfunction syndromes and were not included in the analysis. The most common indications for cardiovascular surgery were degenerative annuloaortic ectasia ( $\mathrm{N}=45$ cases), Marfan syndrome ( $\mathrm{N}=27$ cases), and bicuspid aortic valve $(\mathrm{N}=11$ cases). Eleven patient cases were urgent procedures. Notably, 52 patient cases were concomitant surgical procedures, including trans-aortic stented graft implantation in 15 patient cases; total arch in 14 patient cases; mitral valvuloplasty in 13 patient cases; tricuspid valvuloplasty in 3 patient cases; graft replacement of hemi-arch, coronary artery bypass grafting, or repair of an atrial septal defect in 2 patient cases, each; and mitral valve replacement in 1 patient case (Table 1 ).

Fifty-six $(56,57.7 \%)$ of the patient cases were managed using surgical sealant application to the aortic root suture line, and 41 (46.3\%) did not receive the sealant reinforcement (control group). Selected demographic and medical/surgical data for these 2 patient case groups are summarized in Table 1 . There were no statistically significant between-group differences noted in patient demographics.

The patient cases managed with versus without surgical sealant exhibited a significantly longer CPB time (140.6 \pm 65.24 minutes versus $114.9 \pm 37.60$ minutes; $P=.016)$ and a significantly longer ACC time $(94.8 \pm 35.79$ minutes versus $73.7 \pm 24.96$ minutes; $P=.001)$. There were more cases in the surgical sealant group than the control group (11 versus $5, P=.032$ ) who required deep hypothermic circulatory arrest (DHCA), while the DHCA time between groups showed no significant difference $(27.3 \pm 9.62$ minutes versus $25.6 \pm 8.44$ minutes, $P=.581$ ).

The postoperative bleeding-related outcomes are summarized by the surgical sealant utilization and control group in Table 2. The 48-hour postoperative drainage volume significantly was less in the surgical sealant group $(846.4 \pm 340.33$ $\mathrm{mL}$ ) as compared with the drainage volume recorded in those patient cases who did not have surgical sealant application $(1029.0 \pm 468.58 \mathrm{~mL} ; P=.028)$ (Figure). The surgical sealant group also exhibited a significantly lower utilization of 
Table 1. Selected demographics and medical/surgical parameters, by surgical sealant utilization or control patient case group

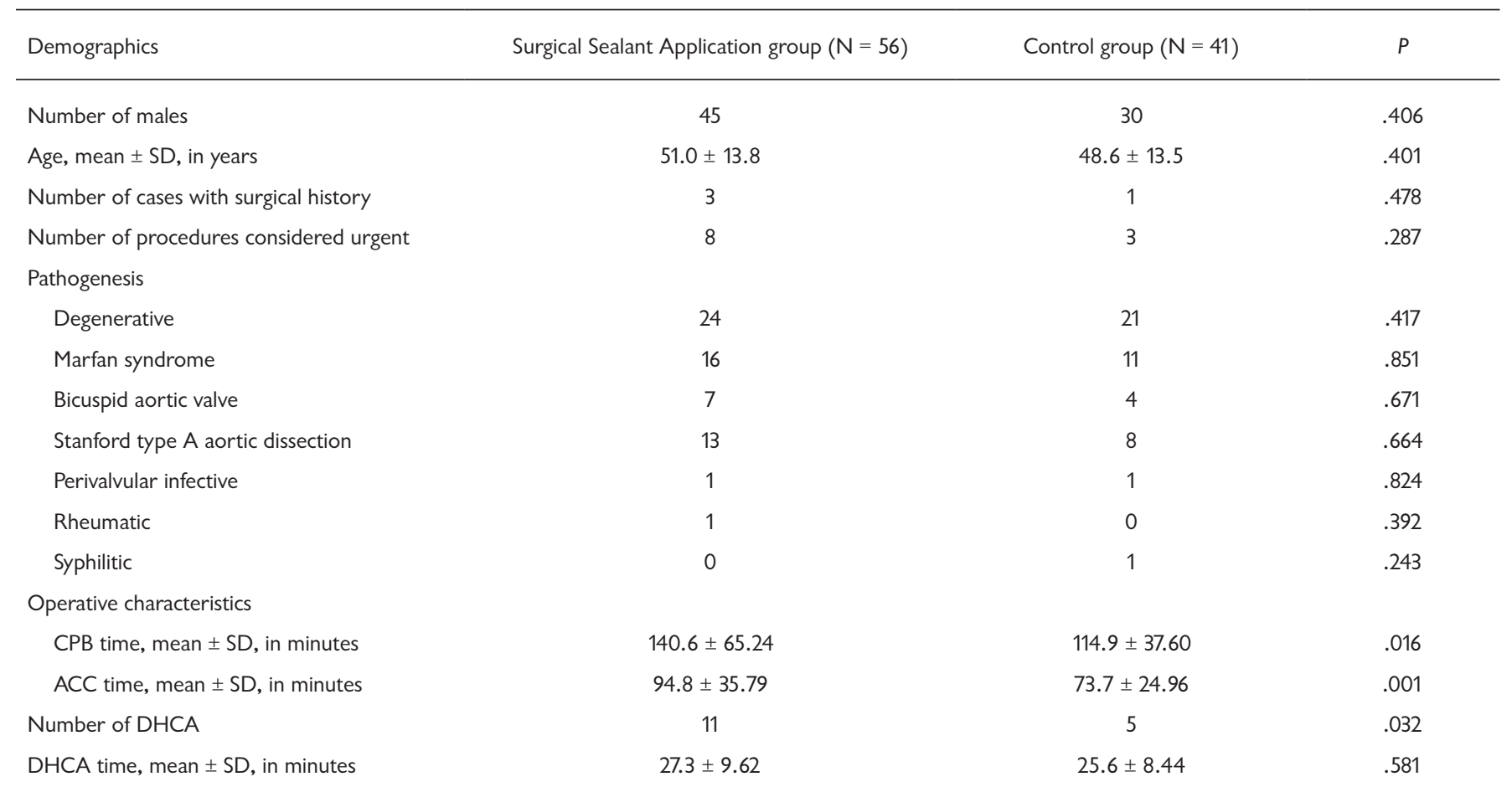

red blood cell and/or fresh frozen plasma transfusion, during both the first 48 postoperative hours as well as during their hospital course. Nearly $45 \%$ of those in the surgical sealant group required no blood transfusion either intraoperatively or postoperatively as compared with less than $15 \%$ in the control group (44.6\% [25/56] versus $14.6 \%$ [6/41]; $P=.002)$. The need for reoperation due to postoperative hemorrhage occurred in a significantly lower proportion of those in the surgical sealant versus the control group $(1.7 \%$ [1/56] versus $12.2 \%[4 / 41] ; P=.036)$. There were no significant betweengroup differences in intubation time or ICU duration ( $P=.636$ and $P=.294$, respectively). When urgent patient cases and those with Stanford type A aortic dissection were excluded, there was a significantly lower ICU duration in the surgical sealant versus the control group $(2.5 \pm 2.27$ days $[\mathrm{N}=43]$ versus $4.7 \pm 5.28$ days $[\mathrm{n}=33] ; P=.017)$. There was no significant difference in postoperative severe infection observed between the two groups $(P=.127)$.

There were no adverse effects reported that were related to the application of surgical sealant in the 56 patient cases reviewed.

\section{DISCUSSION}

In this single-center, retrospective patient case analysis of Bentall procedures, the application of surgical sealant had beneficial effects on postoperative bleeding-related outcomes with no adverse effects. In comparison with suturing alone, the application of surgical sealant significantly reduced the mean postoperative drainage volume when applied onto anastomotic suture lines. Also, the reduction of both requirements for blood transfusion and re-sternotomy for postoperative hemorrhage were observed. A higher proportion of patients who had surgical sealant application required no blood transfusion(s) compared with those without surgical sealant/hemostasis agent utilization. In the full patient-case analysis, there was no significant difference between the surgical sealant application and the control group in intubation time or ICU duration. However, for selective and nondissection cases, there was a significantly lower ICU duration in the surgical sealant application group than in the control group $(P=.017)$. As a result, we may deduce that the application of surgical sealant is associated with improved bleedingrelated outcomes in patients undergoing Bentall procedures. Notably, a longer CPB and ACC time were observed in the surgical sealant group, and this could potentially have a greater benefit from sealant application to aid in coagulation, needle-hole protection. This may have been due to betweengroup differences in condition severity or other hemostasis parameters (e.g., coagulopathy, platelet dysfunction, red cell hemolysis) that were not considered in this retrospective medical/surgical case review. In the surgical sealant group, we did observe a higher proportion of urgent procedures $(14.2 \%$ [8/56] versus $7.3 \%$ [3/41] in the control group) and a greater proportion of aortic dissection cases (23.2\% [13/56] versus $19.5 \%$ [8/41] in the control group).

Urgent surgical case presentation, complex aortic reconstruction, the need for multiple suture lines, prolonged $\mathrm{CPB}$, and occurrence of hypothermia increase the risk for intraoperative and postoperative bleeding and the need for blood product transfusion(s) [Chu 2013]. The increasing 
Table 2. Postoperative bleeding-related outcomes, by surgical sealant utilization or control patient-case group

\begin{tabular}{|c|c|c|c|}
\hline Postoperative drainage, mean $\pm S D$ in $\mathrm{mL}$, during first 48 hours & $846.4 \pm 340.33$ & $1029.0 \pm 468.58$ & .028 \\
\hline During first 48 hours & $2.8 \pm 3.31$ & $4.5 \pm 3.78$ & .022 \\
\hline Total & $3.1 \pm 3.70$ & $4.9 \pm 4.19$ & .027 \\
\hline During first 48 hours & $364.3 \pm 412.72$ & $656.1 \pm 566.59$ & .004 \\
\hline Total $392.9 \pm 451.20$ & $700.0 \pm 579.80$ & 0.004 & \\
\hline Number of patient cases without blood transfusion & 25 & 6 & .002 \\
\hline Number of patient cases undergoing open exploration & 1 & 5 & .036 \\
\hline Patient case intubation time, in hours & $35.7 \pm 63.96$ & $44.4 \pm 115.54$ & .636 \\
\hline
\end{tabular}

emergence of these types of cases along with the shortage of blood donors, the increasing cost of blood-products and transfusion administration, and the potential for transfusionrelated complications have driven greater efforts to minimize bleeding risk and the need for blood transfusion. Advances in cardiac surgical techniques, including the advent of topical hemostatic agents substantially have improved our need for and utilization of blood products. It has been reported that applying surgical sealant (CoSeal) along vascular anastomosis during aortic reconstruction can reduce postoperative drainage loss and transfusion requirements and reduce healthcare costs [Natour 2012]. It is flexible and compliant to allow aorta normal physiologic dilation without contributing to elevated wall stress by stiffening surrounding tissues which may weaken tissue structure and potentiate late pseudoaneurysm formation [Elefteriades 2009; Azadani 2009].

In the performance of Bentall procedures at our hospital in China, we have realized success utilizing the following operative and surgical sealant technique in patients undergoing aortic root reconstruction. The procedure is performed through a median sternotomy and $\mathrm{CPB}$ is instituted with the cannulation of ascending aorta, or femoral artery and bicaval drainage. Right axillary cannulation is performed in patients with aortic dissection, ascending aorta aneurysm extending to the aortic arch, which required deep hypothermia cardiac arrest (DHCA). Simultaneously, anterograde and retrograde intermittent cold hyperkalemic blood cardioplegia is applied for the myocardial protection. DHCA with cerebral perfusion $\left(20^{\circ} \mathrm{C}\right)$ is applied in patients requiring concomitant aortic arch replacement. After cross-clamping the ascending aorta, the aorta is completely transected and removed from the sinotubular junction to $2 \mathrm{~cm}$ from the cross-clamp. The coronary buttons with a $1 \mathrm{~cm}$ diameter cuff of the aortic wall are prepared before the excision of the native leaflets and Valsalva sinuses. A flanged composite graft is prepared in the operating room. After the aortic annulus is measured, we select the tubular graft (Vascuteck®, Terumo Corp., Tokyo,
JPN) 1 or 2 sizes wider than the mechanical prosthetic valve (St. Jude Medical, Inc., St. Paul, MN, USA). The prosthetic valve is inserted into the graft and fixed with a continuous 4-0 polypropylene running suture. The composite valve graft is implanted by attaching the prosthetic valve ring of the handmade composite valved conduit into the aortic annulus with interrupted pledgeted horizontal mattress polyester U-sutures. The coronary buttons are attached to the composite valved conduit end-to-side with a continuous 5-0 polypropylene suture. Then, the distal anastomosis is performed with a running 4-0 polypropylene suture. For the application of surgical sealant, we use CoSeal, which is provided in a kit that contains its two PEG components, and an applicator was utilized. A standard applicator with the volumes of the sealant from 2 to $4 \mathrm{~mL}$ is prepared for aortic reconstruction procedures after the anastomosis had been sutured and before restoring blood circulation to re-expand the graft. Excess blood is aspirated, and all surfaces are blotted or airdried before surgical sealant application. The surgical sealant forms a hydrogel that adheres to tissue and graft materials and covalently bonds to itself. It is applied in a dropwise fashion to enhance mixing, while moving quickly along the anastomotic site. A uniform layer of sealant is applied at $3 \mathrm{~cm}$ to 6 $\mathrm{cm}$ to the treatment site. We overlap the application slightly to ensure complete coverage of the treatment site. Following application, the surgical sealant quickly polymerizes in approximately 5 seconds and reaches full mechanical strength in approximately 1 minute. In our technique, we wait at least 60 seconds before removing the cross-clamp, applying irrigation, blotting with gauze, or touching the sealant.

We believe that the findings of our retrospective patient case review indicate that the use of surgical sealants in Bentall procedures is beneficial to reduce the risk for bleeding and the need for blood product transfusion. Also, we hope that our colleagues benefit from the summary of our surgical technique. We do recognize that these encouraging findings should be interpreted in the light of the limitations 
of a retrospective, single-center study that included a relatively small number of patients and limited details regarding the sites of surgical sealant application. Also, these procedures were performed by seven cardiac surgeons and while they followed a standardized protocol, inherent differences in the experience and surgical techniques exist among these surgeons. Despite these limitations, our study demonstrates the beneficial advantage of the application of surgical sealant in Bentall procedures.

In conclusion, this retrospective case review indicates that the use of surgical sealant is effective in reducing blood loss, transfusion requirements, and re-sternotomy for postoperative hemorrhage in patients undergoing Bentall procedures. The findings of this analysis warrant further investigation in prospective, multicenter studies. Future evaluations of the impact of surgical sealant application should include greater proportions of urgent cases and Stanford type A aortic dissection cases, which prolong the $\mathrm{CPB}$ circuit and enhance hemostasis challenges.

\section{REFERENCES}

Agha RA, Borrelli MR, Vella-Baldacchino M, Thavaygan R, Orgill DP and the STROCSS Group. 2017. The STROCSS Statement: Strengthening the reporting of cohort studies in surgery. Intl J Surg 46:198-202.

Association of Perioperative Registered Nurses (AORN). 2013. Management of surgical hemostasis: An independent study guide. Published 4 Jan 2013. Available at www.slideshare.net/aornsocialmedia/hemostasisstudy-guide. Accessed 12 Dec 2018.

Azadani AN, Matthews PB, Ge L, Shen Y, Jhun CS, Guy TS, Tseng EE. 2009. Mechanical properties of surgical glues used in aortic root replacement. Ann Thorac Surg 87:1154-1160.

Chu MW, Losenno KL, Moore K, Berta D, Hewitt J, Ralley F. 2013. Blood conservation strategies reduce the need for transfusions in ascending and aortic arch surgery. Perfusion 28:315-321.

D'Agostino RS, Jacobs JP, Badhwar V, et al. 2019. The society of thoracic surgeons adult cardiac surgery database: 2019 update on outcomes and quality. Ann Thorac Surg 107:24-32.
Dapunt OE, Easo J, Hölzl P, et al. 2008. Stentless full root bioprosthesis in surgery for complex aortic valve-ascending aortic disease: a single center experience of over 300 patients. Eur J Cardiothorac Surg. 33: $554-559$.

DeAnda A, Elefteriades JA, Hasaniya NW, Lattouf OM, Lazzara RR. 2009. Improving outcomes through the use of surgical sealants for anastomotic sealing during cardiovascular surgery. J Card Surg 24:325-333.

Elefteriades JA. 2009. How I do it: utilization of high-pressure sealants in aortic reconstruction. J Cardiothorac Surg 4:27.

Ferraris VA, Brown JR, Despotis GJ, et al. 2011. Society of Thoracic Surgeons Blood Conservation Guideline Task Force. 2011 Update to the society of thoracic surgeons and the society of cardiovascular anesthesiologists blood conservation clinical practice guidelines. Ann Thorac Surg 91:944-982.

Geissler RG, Rotering H, Buddendick H, et al. 2015. Utilization of blood components in cardiac surgery: a single-centre retrospective analysis with regard to diagnosis-related procedures. Transfus Med Hemother 42:75-82.

Glickman M, Gheissari A, Money S, Martin J, Ballard JL. 2002. CoSeal Multicenter Vascular Surgery Study Group, A polymeric sealant inhibits anastomotic suture hole bleeding more rapidly than gelfoam/thrombin: results of a randomized controlled trial. Arch Surg 137:326-332.

Hagberg RC, Safi HJ, Sabik J, Conte J, Block JE. 2004. Improved intraoperative management of anastomotic bleeding during aortic reconstruction: results of a randomized controlled trial. Am Surg 70:307-311.

Hei F, Zhu DM, Hou XT. 2017. Chinese white paper on cardiac surgery and CPB white paper. Chin J ECC 15:65-67.

Milne AA, Murphy WG, Reading SJ, Ruckley CV. 1996. A randomized trial of fibrin sealant in peripheral vascular surgery. Vox Sang 70:210-212.

Natour E, Suedkamp M, Dapunt OE. 2012. Assessment of the effect on blood loss and transfusion requirements when adding a polyethylene glycol sealant to the anastomotic closure of aortic procedures: a casecontrol analysis of 102 patients undergoing Bentall procedures. J Cardiothorac Surg 7:105.

Robich MP, Koch CG, Johnston DR, et al. 2015. Trends in blood utilization in United States cardiac surgical patients Transfusion 55:805-814.

Velasquez CA, Singh M, Mahmood SU, et al. 2017. The effect of blood transfusion on outcomes in aortic surgery. Int J Angiol 26:135-142. 